\title{
Bergapten exerts inhibitory effects on diabetes-related osteoporosis via the regulation of the PI3K/AKT, JNK/MAPK and NF-кB signaling pathways in osteoprotegerin knockout mice
}

\author{
XUE-JU LI" ${ }^{*}$ ZHE ZHU*, SI-LIN HAN and ZI-LONG ZHANG \\ Department of Orthopaedics, Zaozhuang Municipal Hospital, Zaozhuang, Shandong 277100, P.R. China
}

Received December 20, 2015; Accepted July 8, 2016

DOI: $10.3892 /$ ijmm.2016.2794

\begin{abstract}
Diabetes, as a serious metobolic disorder, poses global threat to human health. It is estimated that over 50 million individuals are already affected by diabetes. Currently, diabetes-related osteoporosis has been a research hotspot due to its high incidence rate in older individuals. Osteoprotegerin, as an important protein for the prevention of osteoporosis, has been proven to be key to the suppression of osteoporosis. Hence, the loss of function of osteoprotegerin may promote the development of osteoporosis. Bergapten, as a natural anti-inflammatory and anti-tumor agent isolated from bergamot essential oil, other citrus essential oils, and grapefruit juice, has been proven to have the ability to attenuate a number of metabolic disorders. In view of these findings, in this study, we used a high-fat diet to construct a mouse model of diabetes-related osteoporosis and a mouse model of diabetesrelated osteoporosis using osteoprotegerin knockout mice. Enzyme-linked immunosorbent assay (ELISA), qPCR, western blot analysis, immunohistochemical assay, H\&E staining, Oil Red O staining, Masson's staining and other biochemical analyses were used to evaluate the related signaling pathways involved in the development of diabetes-related osteoporosis. We also examined the role of osteoprotegerin in the activation of these pathways and in the development of osteoporosis, as well as the protective effects of bergapten against diabetes-related osteoporosis and on the activation of related signaling pathways. Our results revealed that in diabetes-related osteoporosis, the phosphoinositide 3-kinase (PI3K)/AKT, c-Jun N-terminal kinase (JNK)/mitogen-activated protein kinase (MAPK) and
\end{abstract}

Correspondence to: Dr Zhe Zhu, Department of Orthopaedics, Zaozhuang Municipal Hospital, 41 Longtou Road, Zaozhuang, Shandong 277100, P.R. China

E-mail: zhuzhe277100@126.com

*Contributed equally

Key words: diabetes-related osteoporosis, osteoprotegerin, PI3K/ AKT, JNK/MAPK, nuclear factor- $\kappa B$ nuclear factor- $\mathrm{B}(\mathrm{NF}-\kappa \mathrm{B})$ signaling pathways were activated and the expression levels of related indicators were increased. At the same time, osteoprotegerin knockout further promoted the activation of these pathways. By contrast, bergapten exerted effects similar to those of osteoprotegerin. Bergapten exhibited the ability to significantly inhibit RANKL-RANK signaling transduction, and to suppress the activation of the PI3K/AKT, JNK/MAPK and NF- $\kappa B$ signaling pathways, thus protecting trabecular structure and decreasing osteoclastogenic differentiation.

\section{Introduction}

Osteoporosis is a disease where decreased bone strength increases the risk of bone fractures. It is the most common reason for bone fractures among older individuals. According to published data, the National Osteoporosis Foundation (NOF) estimates that as many as 54 million Americans have osteoporosis and decreased bone mass (1-3). Studies have shown that approximately half of the female and one quarter of the male population will suffer from bone fractures due to osteoporosis $(4,5)$. More than $50 \%$ of non-Hispanic Caucasian and Asian women aged 50 years and older have a decreased bone mass, thus increasing their risk for osteoporosis $(6,7)$. A major reason for this is that the female body produces less estrogen after menopause, and estrogen plays an important role in helping to prevent bone loss $(8,9)$. Decreased bone mass poses a serious health concern for individuals worldwide, as it is known that severe osteoporosis is associated with other disorders and diseases. Osteoporosis may occur due to lower than normal peak bone mass and greater than normal bone loss. Bone loss increases after menopause due to lower levels of estrogen $(8,9,10,11)$.

Osteoporosis may also occur due to a number of diseases or treatments, including alcoholism, anorexia, hyperthyroidism, surgical removal of the ovaries and kidney disease (12). Certain medications increase the rate of bone loss, including some anti-seizure medications, chemotherapy, proton pump inhibitors, selective serotonin reuptake inhibitors and steroids (13). Insufficient exercise and smoking are also considered risk factors. Among these factors, diabetes mellitus (DM) is considered an important risk factor for the development of osteoporosis and this has been widely noted 
by researchers $(14,15)$. Diabetes-related osteoporosis (DO) is a general metabolic bone disorder which increases the tendency for fractures due to osteopenia, microstructural changes in bone tissue, decreased bone strength and increased friability, which is one of the main complications of DM affecting the skeletal system. With the increasing morbidity associated with DM, the morbidity associated with DO is markedly increasing, and has become a main cause of mutilation and death in patients with diabetes. DO severely affects the quality of life of patients, and adds heavy economic burden to families and society.

The pathogenesis and prevention of DO has become a worldwide research hotspot (16). In previous studies, the phosphoinositide 3-kinase (PI3K)/AKT, c-Jun N-terminal kinase $(\mathrm{JNK}) /$ mitogen-activated protein kinase (MAPK) and nuclear factor- $\kappa \mathrm{B}(\mathrm{NF}-\kappa \mathrm{B})$ signaling pathways have been proven to be possible targets with which to inhibit the development and progression of DO $(17,18)$. The underlying molecular mechanisms of of action these pathways in DO remain unknown.

Bergapten (BP; $\mathrm{C}_{12} \mathrm{H}_{8} \mathrm{O}_{4}$ ), a natural anti-inflammatory and anti-tumor agent isolated from bergamot essential oil, other citrus essential oils and grapefruit juice, has been used to prevent lipopolysaccharide (LPS)-mediated osteoclast formation, bone resorption and osteoclast survival in vitro (19). Bergapten has also been shown to significantly inhibit the production of pro-inflammatory cytokines (20). However, the effects of Bergapten on diabetes-related osteoporosis are not yet understood.

Hence, in the present study, in an aim to elucidate the mechanisms of action of these pathways in DO, we examined the protective effects of $\mathrm{BP}$ on $\mathrm{DO}$ and investigated the role of the PI3K/AKT, JNK/MAPK and NF- $\kappa \mathrm{B}$ signaling pathways in the protective effects of $\mathrm{BP}$ in osteoprotegerin (OPG) knockout mice.

\section{Materials and methods}

Animals. For the purposes of this study, 6-8-week-old wildtype male C57/B6 mice $(n=102)$ and OPG knockout [OPG(-/-) or OPG-KO; $\mathrm{n}=56$ ] male C57/B6 mice (weighing 25-30 g) were purchased from the Zhejiang University Experimental Animal Center (Zhejiang, China) and kept in a temperature and humidity-controlled environment $\left(25 \pm 2^{\circ} \mathrm{C}, 50 \pm 10 \%\right.$ humidity $)$ with a standard 12-h light/12-h dark cycle with food and water provided in their cages. All experiments involving the use of animals were carried out in accordance with the Institutional Animal Care and Use Committee of the Department of Orthopaedics, Zaozhuang Municipal Hospital. BP (chemical structure shown in Fig. 1A) (CAS:6814421-8, purity $\geq 98 \%$ ) was purchased from Nanjing Biological Technology Co., Ltd. (Nanjing, China) and prepared in phosphate-buffered saline (PBS). The rodent high-fat diet was purchased from Research Diets Inc. (D12492, $60 \mathrm{kcal} \%$ fat; New Brunswick, NJ. USA). The mice were randomly divided into 7 groups as follows (6-10 mice per group): i) normal (wildtype); ii) DO; iii) DO + OPG(-/-); iv) $\mathrm{DO}+10 \mathrm{mg} / \mathrm{kg} /$ day $\mathrm{BP}$; v) $\mathrm{DO}+20 \mathrm{mg} / \mathrm{kg} /$ day $\mathrm{BP}$; vi) $\mathrm{DO}+\mathrm{OPG}(-/-)+10 \mathrm{mg} / \mathrm{kg} /$ day BP; and vii) $\mathrm{DO}+\mathrm{OPG}(-/-)+20 \mathrm{mg} / \mathrm{kg} / \mathrm{day} \mathrm{BP}$. The mice were orally administered BP for 20 weeks. The whole experimental period lasted 24 weeks.
Histological analysis. After the final drug administration, the mice were anesthetized with diethyl ether and mouse blood was harvested by eyeball extraction, 3 mouse liver tissues were collected and stored in $4 \%$ paraformaldehyde. The liver samples obtained were subjected to Oil Red O staining analysis and observed under a light microscope. The Oil Red O staining was performed by Nanjing Biotechnology, Co., Ltd. For bone tissue analysis, Masson's staining, hematoxylin and eosin (H\&E) staining, medical X-rays and immunohistochemical analysis (IHC) were used to evaluate the extent of osteoporosis and the involvement of related key signaling pathways. In brief, formalin-fixed and paraffin-embedded mouse liver specimens were sectioned at $4 \mathrm{~mm}$, stained with H\&E, and used for histopathological examinations. For immunohistochemical staining, the sections were dewaxed and dehydrated. Following antigen retrieval in citrate buffer, we blocked the sections overnight at $4^{\circ} \mathrm{C}$. The sections were then probed with rabbit anti-phosphorylated (p-)JNK (4668), anti-p-AKT (13038), anti-IкB $\alpha$ (4812) and anti-vascular endothelial growth factor (VEGF; 9698) antibodies (Cell Signaling Technolology, Danvers, MA, USA) and an ultra-sensitive immunohistochemistry kit (Maixin, Fuzhou, China) was used. $\mathrm{X}$-rays were obtained using the MutiFocus X-ray imaging system (Faxitron Bioptics LLC, Tucson, AZ, USA). Masson's staining was performed by Biohelper Co. (Nanjing, China) according to a standard protocol.

In order to determine the damage inflicted on the bones of $\mathrm{DO}$ and $\mathrm{DO}+\mathrm{OPG}-\mathrm{KO}$ mice, we also measured the the bone volume fraction (BV/TV), trabecular thickness (Tb.Th) and trabecular number (Tb.N).

Biochemical analysis. The oral glucose tolerance test (OGTT) ( $2 \mathrm{~g} / \mathrm{kg}$ glucose) and insulin tolerance test (ITT) were conducted according to reported methods (21). In brief, OGTTs were performed after final weeks of treatment in mice that had been fasted for $6 \mathrm{~h}$. Glucose was orally administered ( $3 \mathrm{~g} / \mathrm{kg}$ body weight, $660 \mathrm{~g} / \mathrm{l}$ glucose solution) and blood glucose was determined through a glucose meter using $3.5 \mu \mathrm{l}$ of blood collected from the tip of the tail vein before and at the administration of the glucose load (30, 60, 90 and $120 \mathrm{~min})$. Also, the other indicators [lipid metabolism-related indicators, including glucose (GLU), triglycerides (TG), total cholesterol (CHOL), high-density lipoprotein cholesterol (HDLC) and low-density lipoprotein cholesterol (LDLC); and inflammatory cytokines, including interleukin (IL)-2, IL-4, IL-6. IL-1 and IL-1 $\beta$, tumor necrosis factor (TNF)- $\alpha$, interferon (IFN)- $\gamma$, IL-17 and IL-10] shown in Fig. 1D-1F were investigated using respective ELISA kits obtained from Nanjing Jiancheng Bioengineering Institute (Nanjing, China) according to the manufacturer's specifications.

Reverse transcription-quantitative PCR (RT-qPCR) and western blot analysis. After the final drug administration, the mice were anesthetized with diethyl ether and mouse mice blood was harvested by eyeball extraction, and 3 mouse liver (as mentioned above)/visceral adipose/kidney tissues were collected and stored in $4 \%$ paraformaldehyde. The Total RNA isolation system from Omega Bio-Tek, Inc. (Norcross, GA, USA) was used to isolate total RNA from the visceral adipose tissue samples. Briefly, $1 \mu \mathrm{g}$ of total RNA was reverse transcribed using the M-MLV-RT system. The reaction was carried 
Table I. Sequences of primers used for PCR.

\begin{tabular}{ll}
\hline Gene & \multicolumn{1}{c}{ Primer sequences $\left(5^{\prime} \rightarrow 3^{\prime}\right)$} \\
\hline RANKL (forward) & AGTACACCTATCATGGAG \\
RANKL (reverse) & TAGATTGTGAATACTG \\
RANK (forward) & GACAGCCACAATGGGTAGGATGT \\
RANK (reverse) & TCTGTGCCAGATATCTTCTGCTA \\
TAK1 (forward) & TTGCTCCTGCTTCGTTC \\
TAK1 (reverse) & AATAAAGTTTTATGTTGAT \\
NIK(forward) & TCGCAAAGTACGAATC \\
NIK (reverse) & TAGTCCCACAGACAACC \\
IKK $\alpha$ (forward) & GTCCAAAACGACTGTCA \\
IKK $\alpha$ (reverse) & CGGACTCCATACAATCTG \\
IKK $\beta$ (forward) & ATGAGAGTCCCTCGTGTGA \\
IKK $\beta$ (reverse) & TTCTTGTTGCTGACTAACG \\
I BB $\alpha$ (forward) & CCTTAGCCCACTACTTC \\
I BB $\alpha$ (reverse) & CTCCGGCAGGAACTTGAA \\
TRAF6 (forward) & TGCGAATGACGCAGCAA \\
TRAF6 (reverse) & TCCGTCAAGTCGGTCA \\
GAPDH (forward) & AAGGCTGGGGCTCATTTG \\
GAPDH (reverse) & GGGCCATCCACAGTCTTC \\
\hline
\end{tabular}

at $43^{\circ} \mathrm{C}$ for $1 \mathrm{~h}$ and terminated by the inhibition of the enzyme at $70^{\circ} \mathrm{C}$ for $10 \mathrm{~min}$. qPCR were carried out using SYBR-Green kits (Bio-Rad, Hercules, CA, USA) on an ABI PRISM 7900HT detection system (Applied Biosystems, Foster City, CA, USA). Invitrogen (Carlsbad, CA, USA) produced all sequences of the primers for qPCR. The primers used for PCR are listed in Table I.

For western blot analysis, proteins were extracted from the kidney tissues using the T-PER Tissue Protein Extraction Reagent kit (Thermo Fisher Scientific, Inc., Pittsburg, PA, USA) according to the manufacturer's instructions. Protein concentrations were determined using the BCA protein assay kit, and equal amounts of protein were loaded per well on a $10 \%$ sodium dodecyl sulphatepolyacrylamide gel. Subsequently, the proteins were transferred onto polyvinylidene difluoride membranes. The membranes were blocked with Tris-buffered saline containing $0.05 \%$ Tween-20 (TBS-T), supplemented with 5\% skim milk (Sigma, St. Louis, MO, USA) at room temperature for $2 \mathrm{~h}$ on a rotary shaker, followed by washing in TBS-T. The membranes were then incubated with specific primary antibodies diluted in TBST at $4^{\circ} \mathrm{C}$ overnight. The primary polyclonal antibodies used were as follows: rabbit antiGAPDH (1:1000, ab9485), receptor activator of NF- $\kappa$ B (RANK) ligand (RANKL; 1:1000, ab9957), RANK (1:1000, ab200369), transforming growth factor $\beta$-activated kinase 1 (TAK1; $1: 1000$, ab109526), NF-кB-inducing kinase (NIK; 1:1000, ab203568), $\operatorname{IKK} \alpha(1: 1000, a b 32041), \operatorname{IKK} \beta \quad(1: 1000, a b 32135)$, $\mathrm{I} \kappa \mathrm{B} \alpha(\mathrm{ab7217}), \mathrm{p}-\mathrm{I} \kappa \mathrm{B} \alpha(1: 1000, \mathrm{ab} 24783)$, TRAF6 (1:1000, ab33915), AKT (1:1000, ab8805), p-AKT (1:1000, ab38449), PI3K (1:1000, ab151549), p-PI3K (1:1000, ab182651), JNK (1:1000, ab4821), p-JNK (1:1000, ab4821) and p-mammalian target of rapamycin (mTOR; 1:1000, ab109268) (all from
Abcam, USA). diluted in TBST, were incubated with the membranes at $4^{\circ} \mathrm{C}$ overnight. Subsequently, the membranes were washed with TBS-T followed by incubation with the peroxidase-conjugated secondary antibody [anti-rabbit antibody (ab191866); Abcam] at room temperature for $1 \mathrm{~h}$. The immunoactive proteins were detected by using an enhanced chemiluminescence western blotting detection kit. Western blot bands were observed using the GE Healthcare ECL Western Blotting Analysis system (GE Healthcare Life Sciences, Pittsburgh, PA, USA) and exposed to X-ray film (Kodak).

Statistical analysis. Data are presented as the means \pm SEM. Differences between the treatment groups and the controls were compared using GraphPad Prism software (version 6.0; GraphPad Software, San Diego, CA, USA) with a one-way ANOVA and Dunn's least significant difference tests or the Student's t-test. Differences between groups were considered significant at a vaue of $\mathrm{p}<0.05$. The bars in the figures indicate the means $\pm \operatorname{SEM}(\mathrm{n}=10$ mice per group).

\section{Results}

Anti-inflammatory effects of BP on DO-induced systemic inflammation and disorders in DO or OPG-KO mice. In the present study, using a mouse model of DO induced by a highfat diet, we investigated the inflammatory response induced by diabetes and the anti-inflammatory effects of BP on inflammatory cytokine production. As shown in Fig. 1B and C, OGTT and ITT tests revealed that a high-fat diet increased glucose levels in serum and promoted the development of insulin resistance. The OPG-KO mice exhibited higher glucose levels among most of the groups and compared to the normal control group. We also examined the levels of lipid metabolismrelated indicators in each treatment group to examine the effects of OPG-KO and BP treatment on metabolic disorders caused by a high-fat diet. Our results revealed that the levels of lipid metabolism-related indicators, including GLU, TG, CHOL and LDLC were significantly increased in the DO and $\mathrm{DO}+\mathrm{OPG}-\mathrm{KO}$ groups, compared with the BP treatment group and the normal control (Fig. 1D). In addition, the high-fat dietinduced production of inflammatory cytokines was examined by ELISA. Our results revealed that a high-fat diet markedly increased the inflammatory responses and cytokine formation and release (Fig. 1E and F). However, treatment with BP attenuated the increase in the inflammatory response. In addition, we used H\&E staining to examine the effects of a high-fat diet on liver tissue. The shown in Fig. $1 \mathrm{G}$ indicated that a high-fat diet promoted lipid accumulation in the liver in the DO and $\mathrm{DO}+\mathrm{OPG}-\mathrm{KO}$ group; this accumulation was inhibited by treatment with BP (10 and $20 \mathrm{mg} / \mathrm{kg})$. Our data indicated that DO and DO in conjuncion with OPG-KO and with a high-fat diet cause systemic inflammation and disorders, and that these negative effects can be significantly suppressed by treatment with BP.

Effects of OPG knockout and BP on DO. We then examined the effects of OPG knockout and BP on diabetes-related osteoporosis. As shown in Fig. 2A-C, the BV/TV, Tb.Th and Tb.N were tested to evaluate the damage inflicted on bone in DO and $\mathrm{DO}+\mathrm{OPG}-\mathrm{KO}$. The BV/TV, Tb.Th and Tb.N of the mice in the 
A

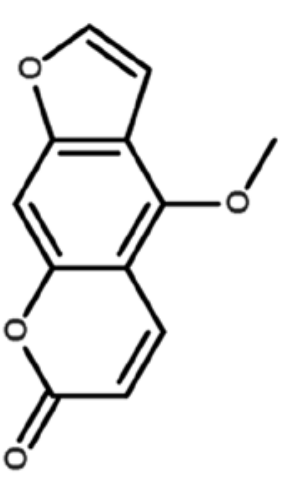

B

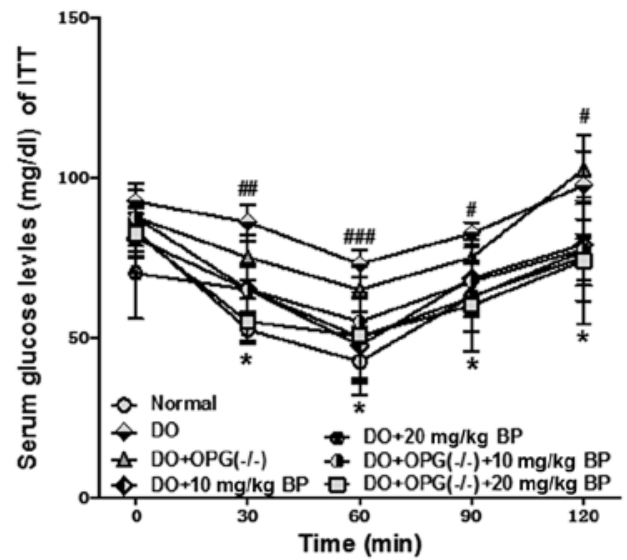

C

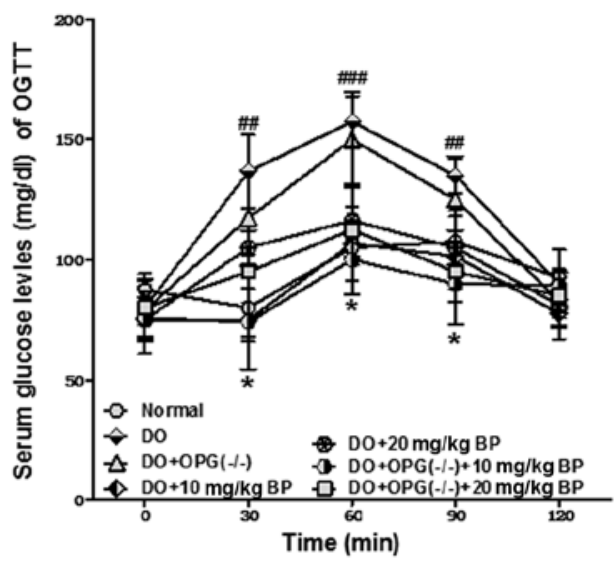

G
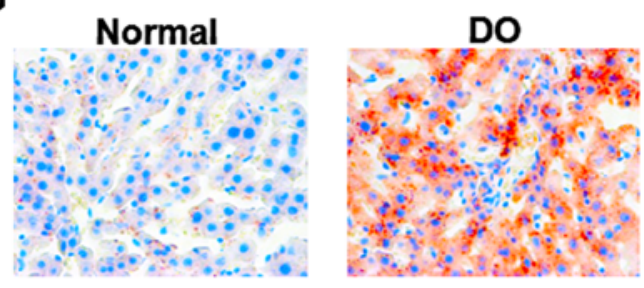

DO+OPG(-I-)

DO+10 mg/kg BP

E

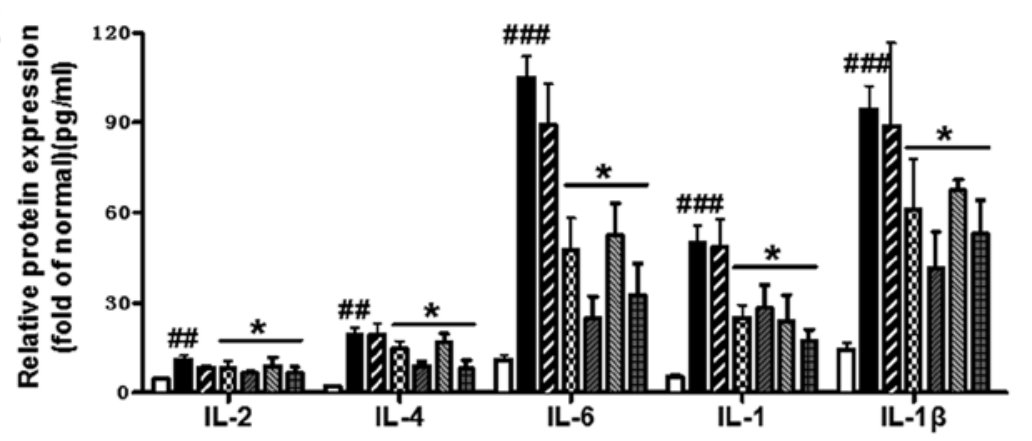

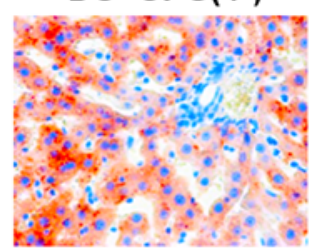

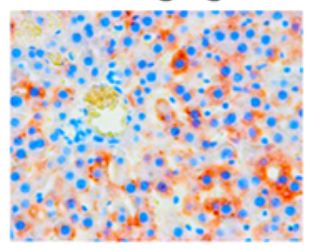

DO+OPG(-l-)+10 $\mathrm{mg} / \mathrm{kg}$ BP
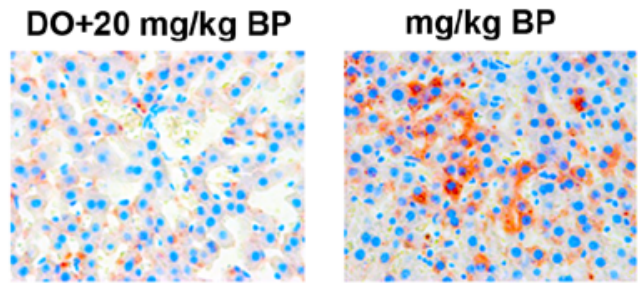

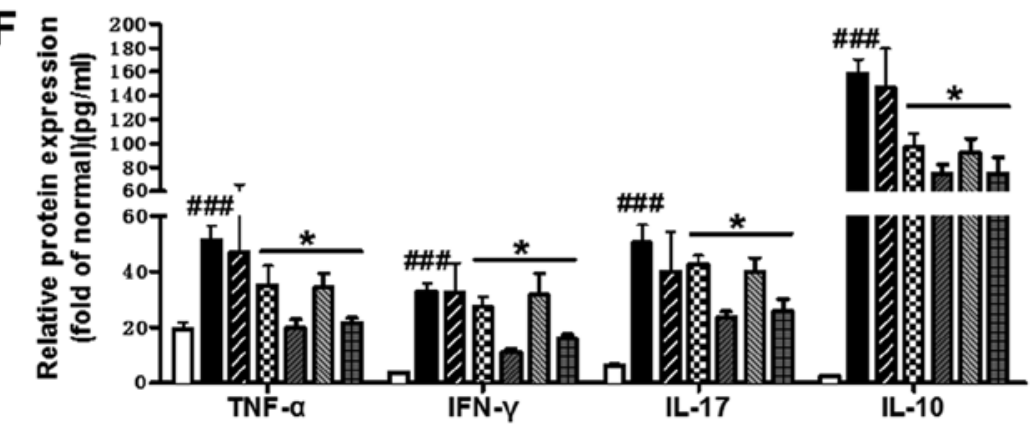

$\mathrm{mg} / \mathrm{kg}$ BP

Figure 1. Bergapten suppresses diabetes mellitus-induced systemic inflammtion and metabolic disorders in mice with diabetes-related osteoporosis (DO) and mice with DO and osteoprotegerin (OPG)-KO. (A) Chemical structure of bergapten. (B) Insulin tolerance test (ITT) for serum glucose levels. (C) Oral glucose tolerance test (OGTT) for serum glucose levels. (D) Biochemical analysis of lipid metabolic indicators. (E) ELISA for interleukin (IL)-2, IL-4, IL-6, IL-1 and IL-1 $\beta$ protein expression. (F) ELISA for tumor necrosis factor- $\alpha$ (TNF- $\alpha$ ), interferon (IFN)- $\gamma$, IL-17 and IL-10 protein expression. (G) Oil Red O staining for analysis of liver tissue of mice fed a high-fat diet. ${ }^{\#} \mathrm{p}<0.05,{ }^{\# \#} \mathrm{p}<0.01,{ }^{\# \# \#} \mathrm{p}<0.001$ vs. normal group; ${ }^{*} \mathrm{p}<0.05$, vs. DO and DO + OPG(-/-) group.

$\mathrm{DO}+\mathrm{OPG}-\mathrm{KO}$ groups were significantly decreased compared with those of the normal group. However, treatment with BP atteuated these negative effects in a dose-independent manner. In addition, X-ray scanning (Fig. 2D) revealed that bone mineral density in the mice in the DO and DO + OPG-KO groups was decreased and bone loss was more severe compared with the normal group. In the DO + OPG-KO group, this was even more severe. These data indicate that OPG is important for protection 

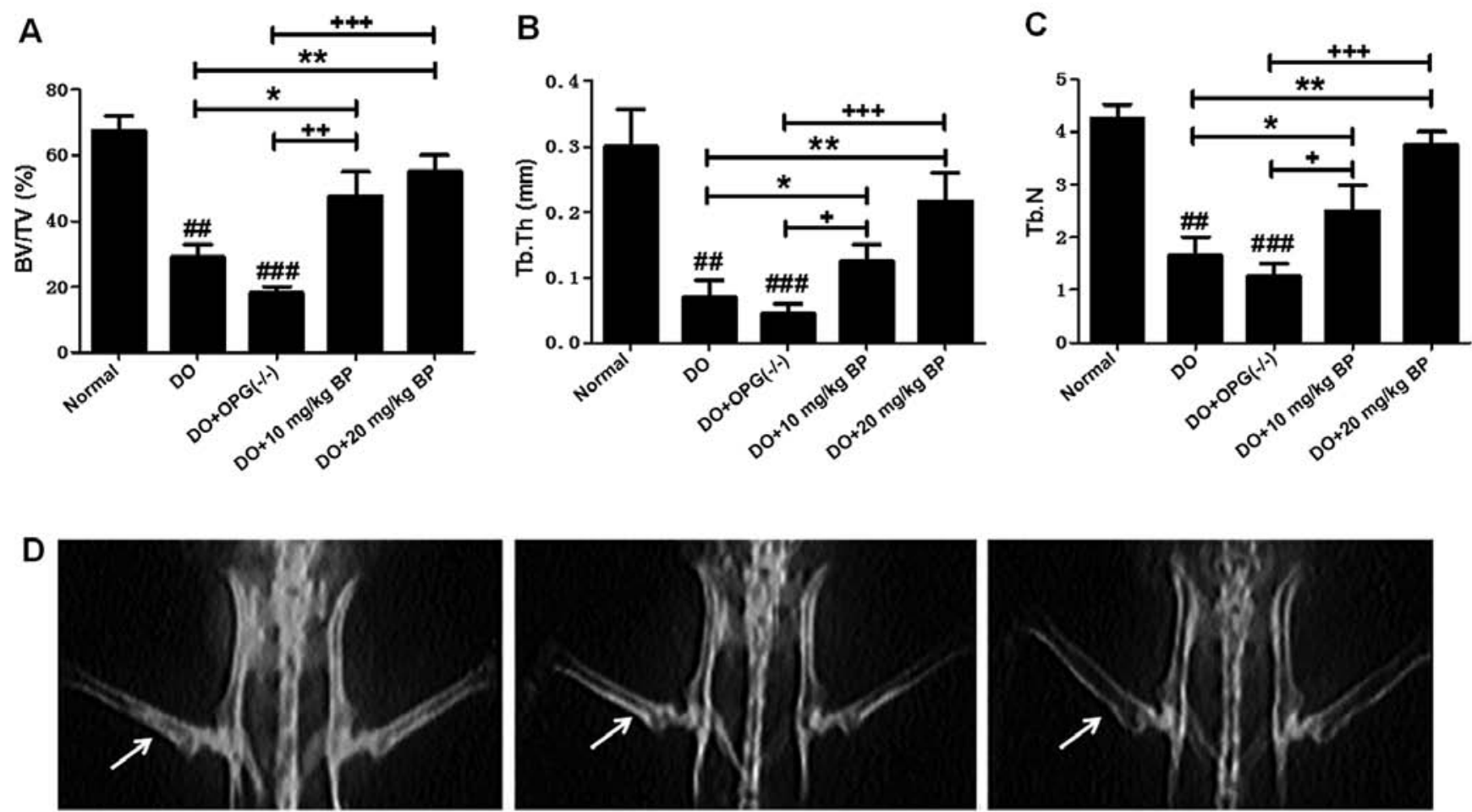

Normal

DO

DO+OPG(-I-)

Figure 2. Effects of osteoprotegerin (OPG) knockout on diabetes-related osteoporosis (DO). (A) Analysis of bone volume fraction (BV/TV) in mice in the normal, $\mathrm{DO}, \mathrm{DO}+\mathrm{OPG}-\mathrm{KO}, \mathrm{DO}+10 \mathrm{mg} / \mathrm{kg}$ bergapten (BP) and DO $+20 \mathrm{mg} / \mathrm{kg}$ BP groups. (B) Analysis of trabecular thickness (Tb.Th) in mice in the normal, DO, $\mathrm{DO}+$ OPG-KO, DO $+10 \mathrm{mg} / \mathrm{kg}$ BP and DO $+20 \mathrm{mg} / \mathrm{kg}$ BP groups. (C) Analysis of trabecular number (Tb.N) in mice in the normal, DO, DO + OPG-KO, $\mathrm{DO}+10 \mathrm{mg} / \mathrm{kg}$ BP and DO $+20 \mathrm{mg} / \mathrm{kg}$ BP groups. ${ }^{\# \#} \mathrm{p}<0.01$ and ${ }^{\# \# \#} \mathrm{p}<0.001$ vs. normal group; ${ }^{*} \mathrm{p}<0.05 ;{ }^{+} \mathrm{p}<0.05,{ }^{++} \mathrm{p}<0.01$ and ${ }^{+++} \mathrm{p}<0.001$. (D) $\mathrm{X}$-ray scanning for determining bone mineral density. The arrows in the images indicate the bone mineral density.

against osteoporosis and that $\mathrm{BP}$ exerts protective effects against DO. Furthermore, H\&E and Masson's staining (Fig. 3A-C) were used to further examine the bone injury assoicated with DO. Our results revealed that in the DO and DO + OPG-KO groups, the trabecular number was decreased and the trabecular bone became thin and there was a decline in trabecular structure compared to the normal group. These effects were more evident in the OPG-KO mice. The bone structure of mice in the DO and $\mathrm{DO}+$ OPG-KO groups was more fragile and chaotic, compared to the normal group. The bone collagen structure of mice in the DO and DO + OPG-KO groups was significantly reduced compared to the normal group. These effects were more evident in the OPG-KO mice. However, treatment with BP attenuated these effects. These data indicate that OPG gene knockout aggravates osteoporosis, and that BP significantly suppresses osteoporosis.

Inhibitory effects of BP on the activation of DO-related signaling pathways. As we mentioned above, in high-fat diet-induced DO, the PI3K/AKT, JNK/MAPK and NF- $\kappa B$ signaling pathways have been proven to be a possible target for the inhibition of the development and progression of DO. However, the underlying molecular mechanisms of action of these pathways in DO remain unknown. Thus in this regard, we used IHC to determine the experssion of p-AKT, p-JNK and the key factor of NF- $\mathrm{KB}, \mathrm{I} \kappa \mathrm{B} \alpha$, the mice in the normal, DO, $\mathrm{DO}+\mathrm{OPG}-\mathrm{KO}$ and $\mathrm{DO}+10 / 20 \mathrm{mg} / \mathrm{kg}$ BP groups. As shown in Fig. 4A, the expression of p-AKT, p-JNK and IкB $\alpha$ of NF- $\kappa B$ in the $\mathrm{DO}$ and $\mathrm{DO}+\mathrm{OPG}-\mathrm{KO}$ groups was significantly higher compared with normal and BP treatment groups. In particular, when comparing the DO group with the DO + OPG-KO group, the DO + OPG-KO group exhibited a higher expressoin of p-AKT, p-JNK and IкB $\alpha$ than the DO group, which indicates that OPG has a protective effect against osteoporosis and that BP has a similar effect to OPG in attenuating DO. In addition, DO promoted the activation of the PI3K/AKT, JNK/MAPK and NF- $\mathrm{KB}$ signaling pathways, enhancing osteoporosis. As shown in Fig. 4B, VEGF experssion was higher the DO and $\mathrm{DO}+\mathrm{OPG}-\mathrm{KO}$ groups compared with the normal group, indicating that greater bone loss occurred in the DO and OPG-KO mice, and this was suppressed by treatment with BP.

OPG knockout promotes the activation of the PI3K/AKT, $J N K / M A P K$ and $N F-\kappa B$ signaling pathways in mice with DO. We then used qPCR and western blot analysis to examine the expression of related indicators of the PI3K/AKT, JNK/ MAPK and NF- $\mathrm{KB}$ signaling pathways in OPG-KO mice. As shown in Fig. 5A-H, the mRNA expression levels of RANKL, RANK, TAK1, NIK, IKK $\alpha$, IKK $\beta$, IкB $\alpha$ and TRAF6 were significantly increased in the OPG-KO group compared to the normal group. In addition, the western blot analysis further indicated that the protein expression levels of RANKL, RANK, TAK1, NIK, IKK $\alpha$, IKK $\beta$, I $\mathrm{B} B \alpha$ and TRAF6 also were further markedly upregulated in the DO + OPG-KO group, at almost higher levels than the DO group (Fig. 5I). This indicates that OPG-KO significantly enhances the activation of the NF- $\mathrm{KB}$ 

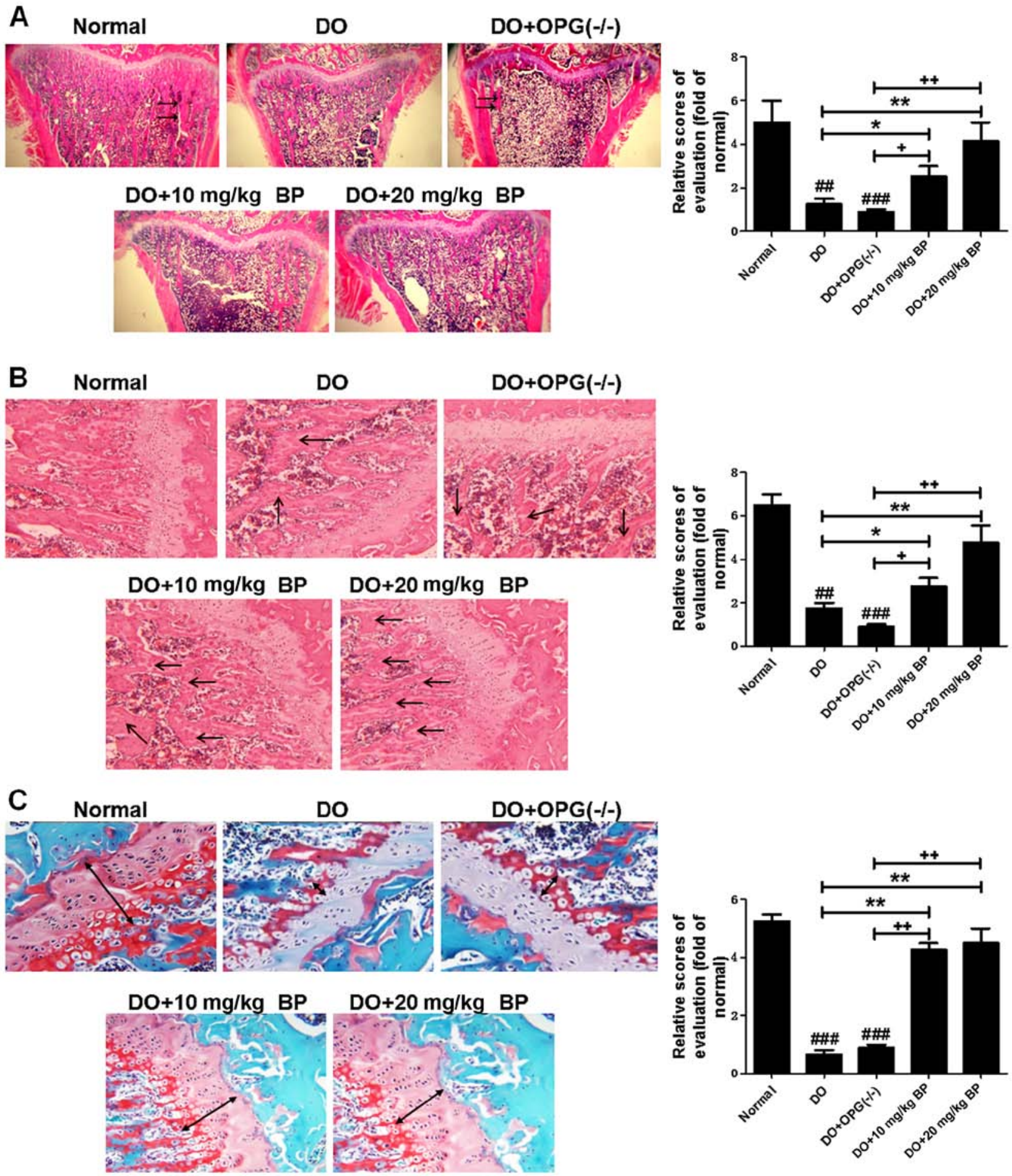

Figure 3. Analysis of bone structure in mice with high-fat diet-induced diabetes-related osteoporosis (DO). (A) Masson's staining for the analysis of trabecular bone. Arrows indicate the trabecular number. (B) Hematoxylin and eosin (H\&E) staining for analysis of bone structure. Arrows indicate the bone structure. (C) Masson's staining for analysis of bone collagen structure. Arrows indicate the bone collagen structure. ${ }^{\# \#} \mathrm{p}<0.01$ and ${ }^{\# \# \#} \mathrm{p}<0.001 \mathrm{vs}$. normal group; ${ }^{*} \mathrm{p}<0.05 ;{ }^{+} \mathrm{p}<0.05$ and ${ }^{++} \mathrm{p}<0.01$.

signaling pathway, thus also promoting the development of DO. Moreover, we also examined the role of the JNK pathway in DO. As shown in Fig. 6, the JNK pathway was activated in the OPG-KO and DO groups. The levels of p-JNK1/2 were significantly increased in the DO + OPG-KO group compared with the normal group, which demonstrates that the JNK pathway is indirectly or directly involved in the development and progression of DO. In addition, we further investigated the role of the AKT signaling pathway in the development of osteoporosis. The data shown in Fig. 7 indicated that the AKT pathway was activated in the OPG-KO mice. The levels of p-AKT and p-mTOR were significantly increased compared 

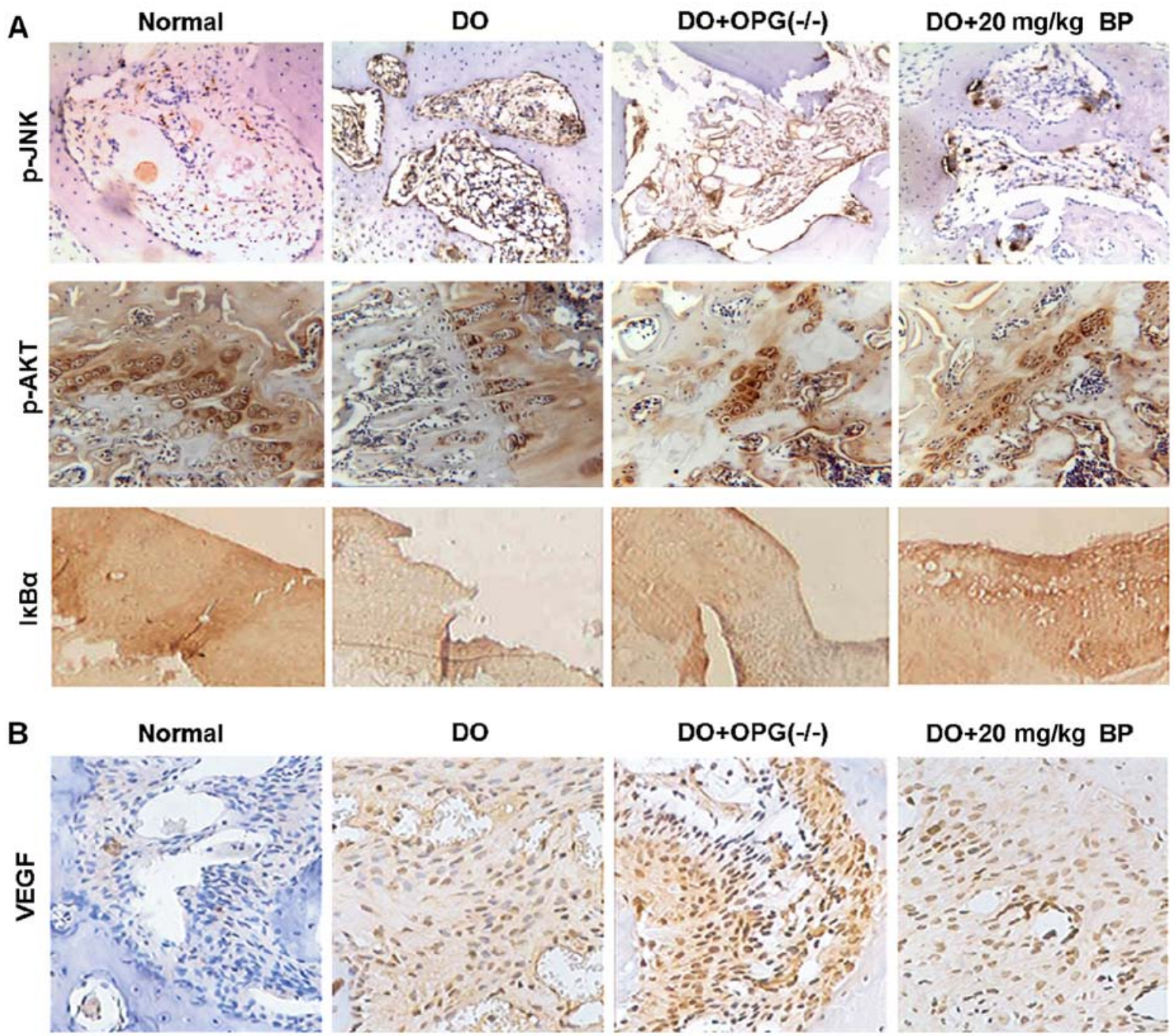

Figure 4. Immunohistochemical analysis for the expression of PI3K/AKT, JNK/MAPK, NF- $\mathrm{KB}$ and VEGF expression in mice with high-fat diet-induced diabetes-related osteoporosis (DO). (A) Levels of phosphorylated JNK, AKT and IאB $\alpha$ expression in mice in the normal, DO, DO + OPG-KO and DO + $20 \mathrm{mg} / \mathrm{kg}$ bergapten (BP).groups (B) VEGF protein expression in mice in normal, DO, DO + OPG-KO and DO + $20 \mathrm{mg} / \mathrm{kg}$ BP groups.

to the normal group. These data indicate that the PI3K/AKT, JNK/MAPK and NF- $\mathrm{KB}$ signaling pathways are indirectly or directly involved in the development of DO.

$B P$ suppresses the activation of the PI3K/AKT, JNK/MAPK and $N F-\kappa B$ signaling pathways in mice with $D O$. We examined the suppressive effects of BP on the activation of the PI3K/ AKT, JNK/MAPK and NF- $\mathrm{KB}$ signaling pathways in our mouse model of DO. As shown in Fig. 8A, scanning electron microscopy revealed that treatment with BP (10 and $20 \mathrm{mg} /$ $\mathrm{kg}$ ) attenuated the development of DO, as evidenced by less surface bone damage in the BP-treated groups compared with the DO + OPG-KO group. Osteoclast activation was enhanced in the DO + OPG-KO group and was significantly inhibited by BP (Fig. 8A). Western blot analysis demonstrated that the levels of related indicators of the PI3K/AKT, JNK/MAPK and NF- $\kappa B$ signaling pathways were significantly suppressed by $\mathrm{BP}$, compared to the DO + OPG-KO group. The inhibitory effects of BP on the levels of these indicators were observed in a dose-independent manner. These data suggest that BP is capable of inhibiting the activation of the PI3K/AKT, JNK/ MAPK and NF- $\mathrm{KB}$ signaling pathways.

\section{Discussion}

Diabetes is regarded as a global threat to human health. Diabetes is a metabolic disorder which causes a dysfunction in the production of insulin, a hormone that is required to convert sugar, starches and other foods into energy. According to published data, it is estimated that $>50$ million individuals are already affected by diabetes, and that 11.6 million diabetics are not aware of the existing disease (1-4). In our current understanding, there are 3 main types of diabetes which have been confirmed: type 1 diabetes, type 2 diabetes and gestational diabetes. Diabetes is the primary reason for adult blindness, end-stage renal disease (ESRD), gangrene and amputations (22-25). Being overweight, lack of exercise, family history and stress increase the likelihood of developing diabetes. Constantly high blood sugar levels lead to kidney failure, cardiovascular problems and neuropathy $(26,27)$. 
A

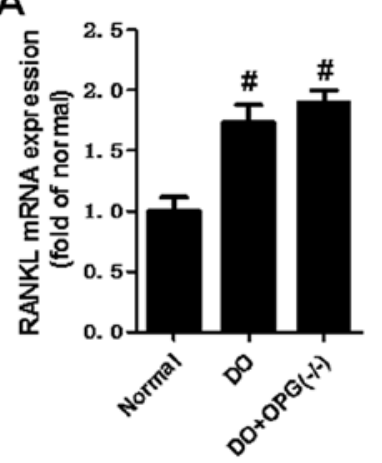

$\mathbf{E}$

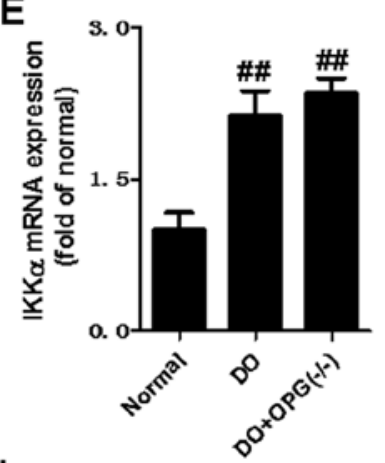

I

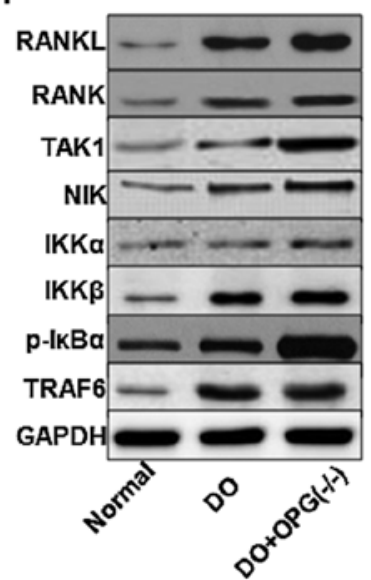

B

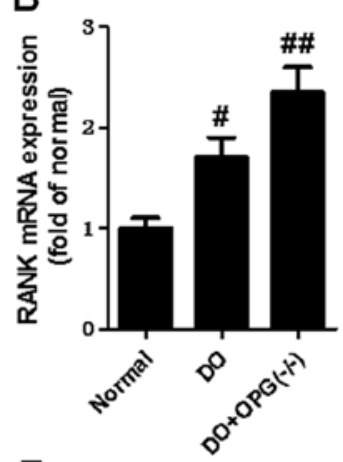

F

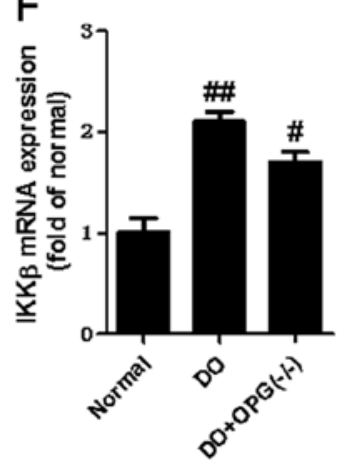

C
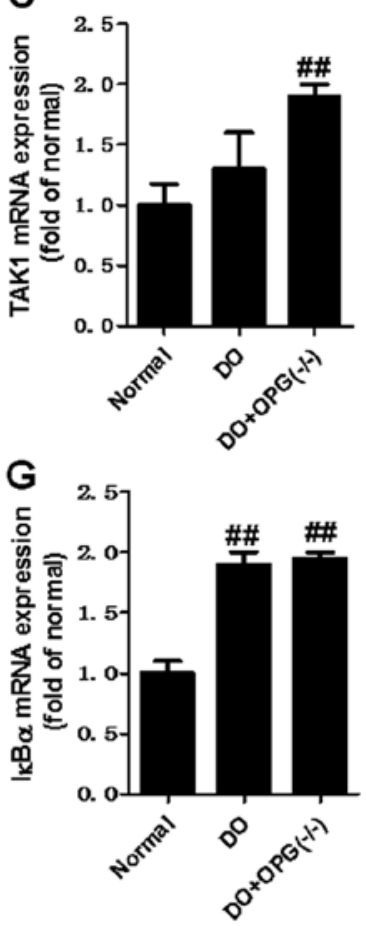

D
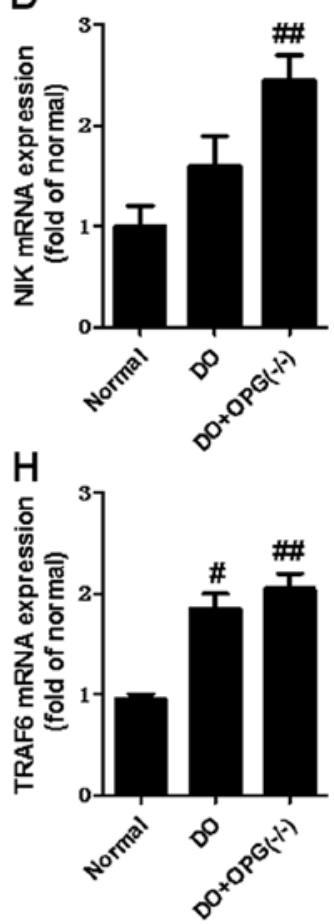

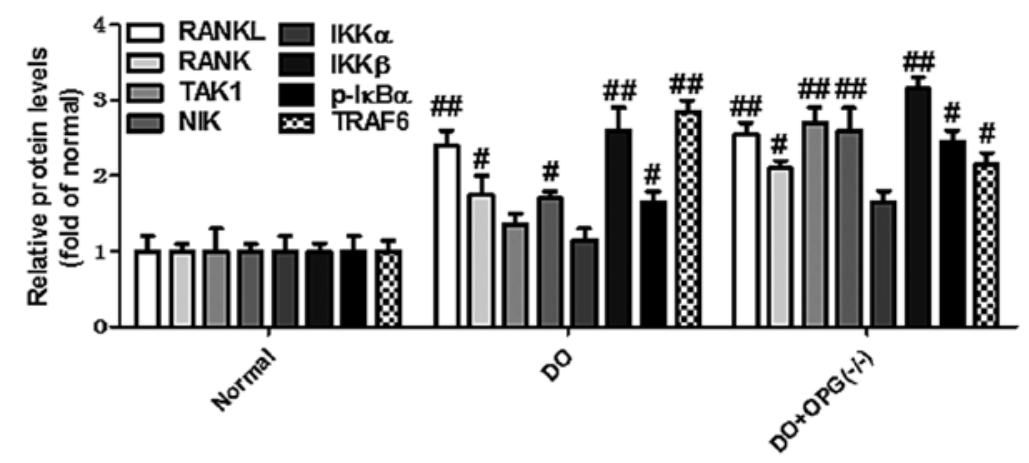

Figure 5. Osteoprotegerin (OPG) knockout promotes the activation of the nuclear factor- $\kappa \mathrm{B}$ (NF- $\kappa \mathrm{B})$ signaling pathway in mice with high-fat diet-induced diabetes-related osteoporosis (DO) and with DO and OPG-KO. (A-H) qPCR of the mRNA expression levels of RANKL, RANK, TAK1, NIK, IKK $\alpha$, IKK $\beta$, $\mathrm{I} \kappa \mathrm{B} \alpha$ and TRAF6. (I) Western blot analysis of the protein expression of related indicators of the NF- $\kappa \mathrm{B}$ signaling pathway. ${ }^{\#} \mathrm{p}<0.05$ and ${ }^{\# \#} \mathrm{p}<0.01 \mathrm{vs.}$ normal group.

A

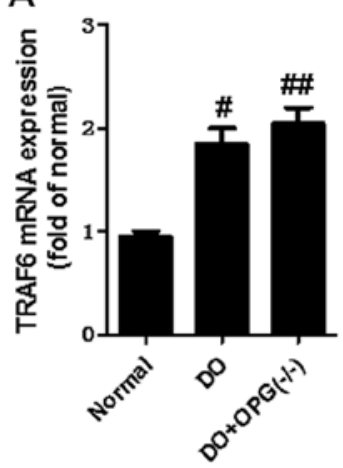

B

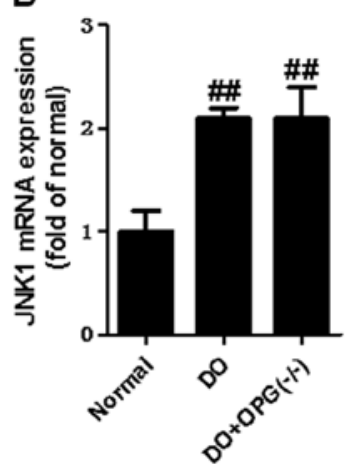

C

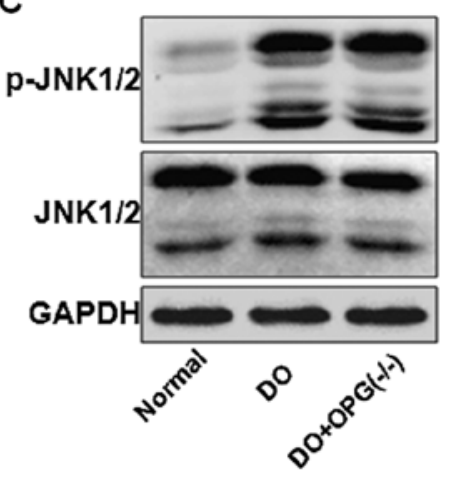

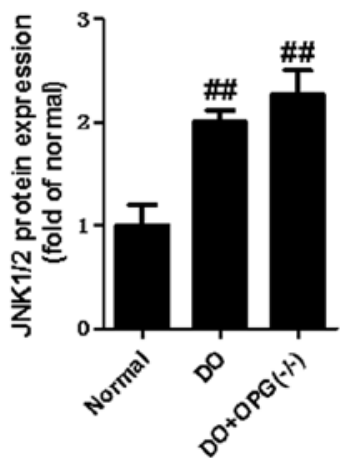

Figure 6. Osteoprotegerin (OPG) knockout promotes the activatio of the JNK/MAPK signaling pathway in mice with high-fat diet-induced diabetes-related osteoporosis (DO) and with DO and OPG-KO. (A and B) qPCR of the mRNA expression levels of TRAF6 and JNK1. (C) Western blot analysis of the protein expression of $\mathrm{JNK} 1 / 2$. ${ }^{\#} \mathrm{p}<0.05$ and ${ }^{\# \#} \mathrm{p}<0.01$ vs. normal group. 
A

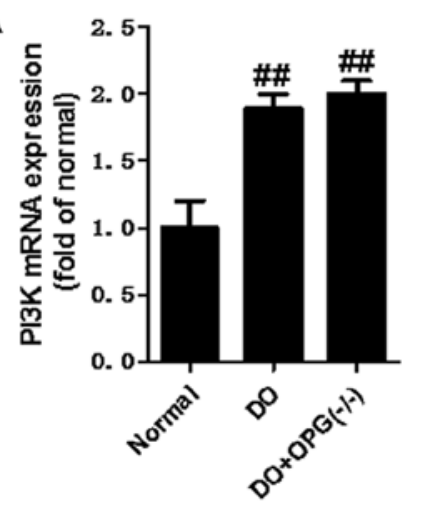

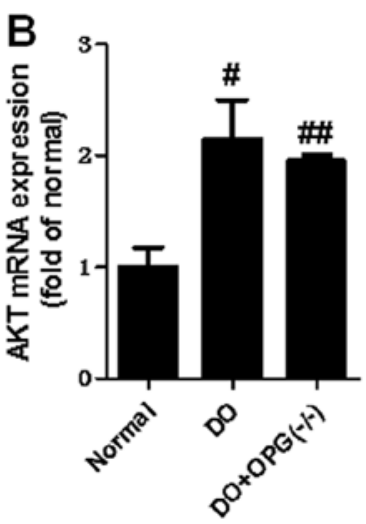

C

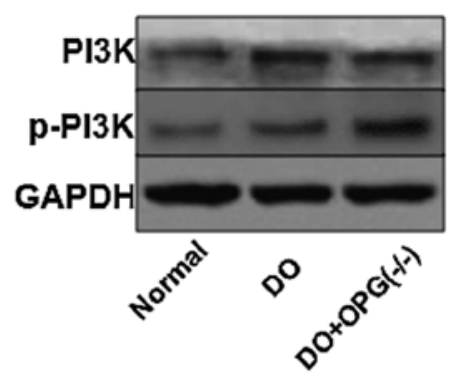

D

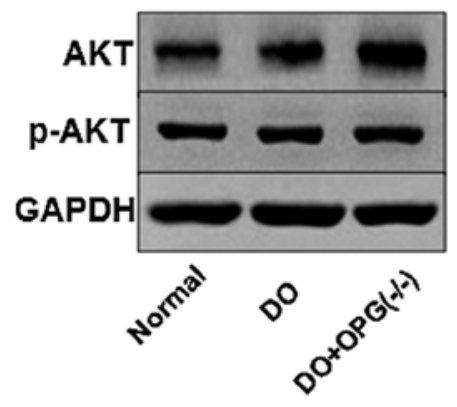

E

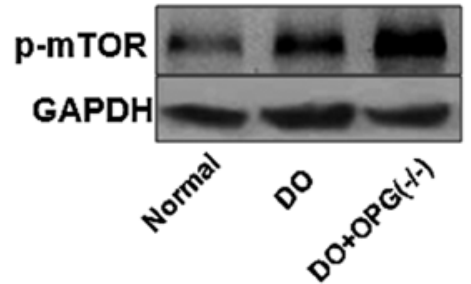

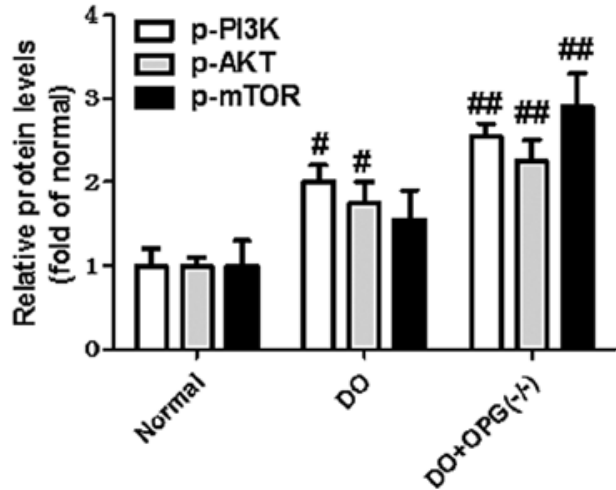

Figure 7. Osteoprotegerin (OPG) knockout promotes the activation of the AKT/mTOR signaling pathway in mice with high-fat diet-induced diabetes-related osteoporosis (DO) and with DO and OPG-KO. (A and B) qPCR of the mRNA expression levels of PI3K and AKT. (C-E) Western blot analysis of the protein expression of AKT/mTOR. ${ }^{\#} \mathrm{p}<0.05$ and ${ }^{\# \#} \mathrm{p}<0.01$ vs. normal group.

Patients with diabetes are 4-fold more likely to suffer from coronary heart disease and stroke. In addition, gestational diabetes is more dangerous for pregnant women and their fetus (28). However, more seriously, in recent years, global researchers have paid more attention to diabetes-related disorders $(29,30)$. At present, there is increasing concern about the many acute and chronic complications of diabetes, among which the association between diabetes and osteoporosis is attracting more attention. DO is a systemic metabolic disease affecting the bone and has an intricate and complex pathogenesis.

DO easily leads to fracture, has a high disability rate and is a serious threat to human health. It has been found that diabetes affects bone metabolism through various mechanisms, including insulin dysfunction, high blood glucose, increased accumulation of advanced glycation end products and microangiopathy $(31,32)$. OPG as an important protein for the preventation of osteoporosis and has been proven to be key to suppressing the development of DO. In this regard, in this study, we used a mouse model of DO a model of DO with OPG knockout mice to investigate the related signaling pathways involved in the development and progression of osteoporosis. In addition, BP as a natural anticancer and antiinflammatory agent isolated from bergamot essential oil, other citrus essential oils and grapefruit juice, was used to examine its protective effects against DO and whether it has a similar effect to OPG in inhibiting osteoporosis.
The PI3K/AKT and related pathways play an important role in internalizing the effects of external growth factors and membrane tyrosine kinases. As shown in Fig. 9, in DO, many downstream indicators and pathways are activated, such as NF- $\mathrm{B}, \mathrm{mTOR}$, Bad and FKHR. The activation of PI3K/AKT further enhances inflammatory cyokine expression and signaling pathway transduction, particularly regarding $\mathrm{NF}-\kappa \mathrm{B}$. Our data found that DO upregulate the level of phosphorylated AKT and activates the downstream mTOR pathway, which promotes the development of osteoporosis. In addition, OPG-KO significantly enhanced the transduction between RANKL and RANK, further activating the AKT pathway. On the one hand, activated AKT further enhances the phosphorylated NF- $\kappa \mathrm{B}$ level, leading to the increased production and and release of inflammatory cytokines. On the other hand, our results revealed that OPG-KO in DO significantly and directly increases the level of phosphorylated NF- $\kappa B$ through $\mathrm{NIK} / \mathrm{IKK} / \mathrm{I} \kappa \mathrm{B} \alpha$ to promote nuclear transduction. Thus, through these two mechanisms, phosphorylated $N F-\kappa B$ is indirectly or directly activated and this causes osteoclastogenic differentiation and loss of ossein, aggravating trabecular bone injury. Furthermore, JNK, as the major pathway in the MAPK family, has been proven to be involved in the development and progression of various diseases $(33,34)$. In this study, our data demonstrated that the MAPK/JNK signaling pathway is involved in the development of osteoporosis. It enhances c-Jun epxression and promotes osteoclastogenic differentiation, in 
A
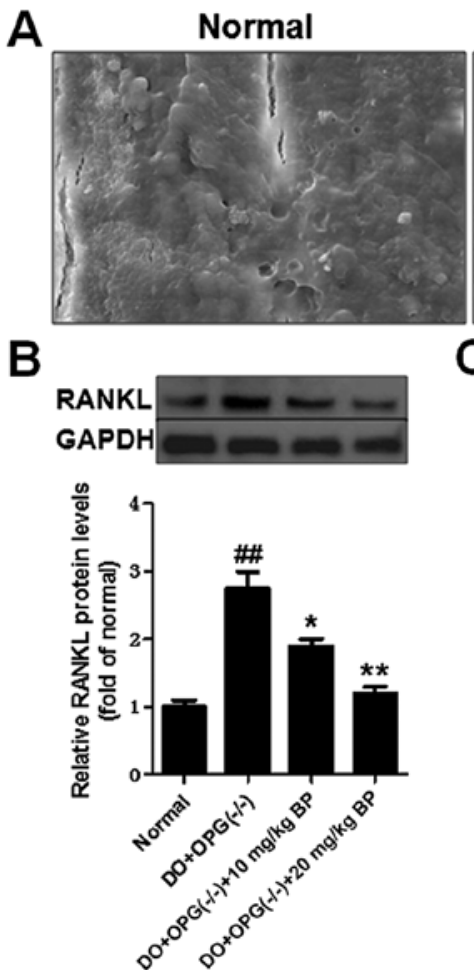

F
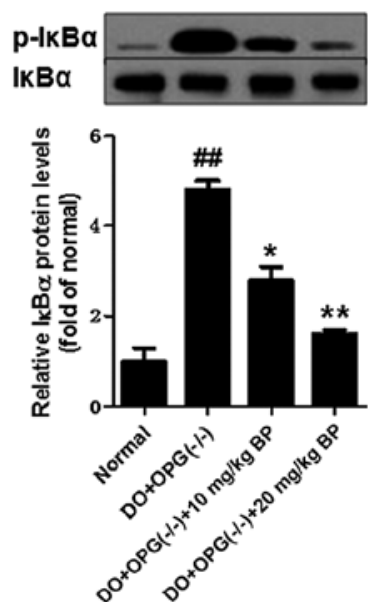

$J$
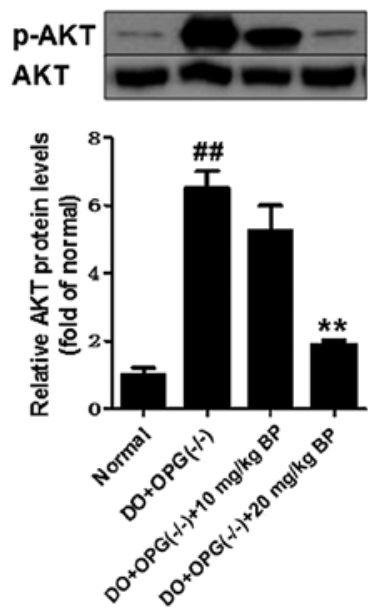

DO+OPG(-l-)

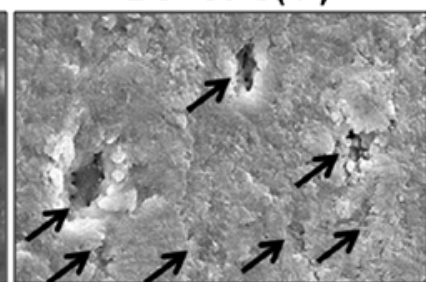

C
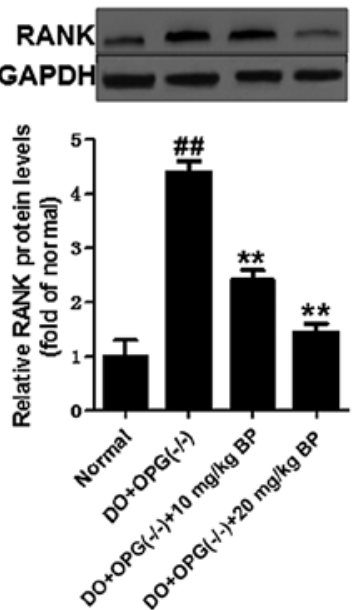

G
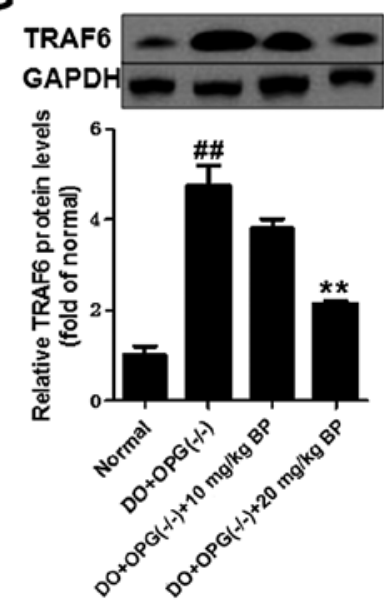

K
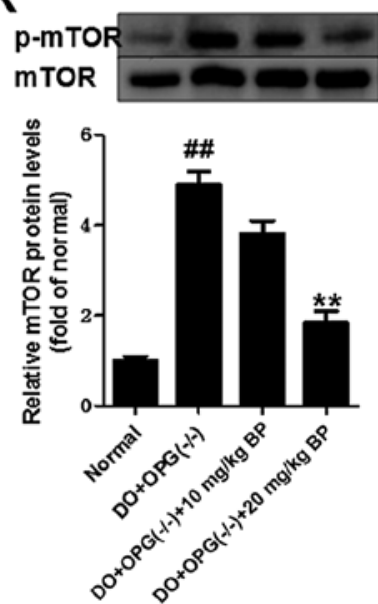

$\mathrm{DO}+10 \mathrm{mg} / \mathrm{kg} \mathrm{BP}$

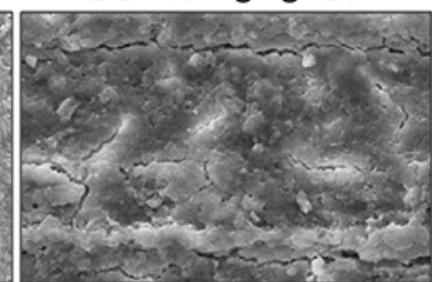

D

TAK1

GAPDH

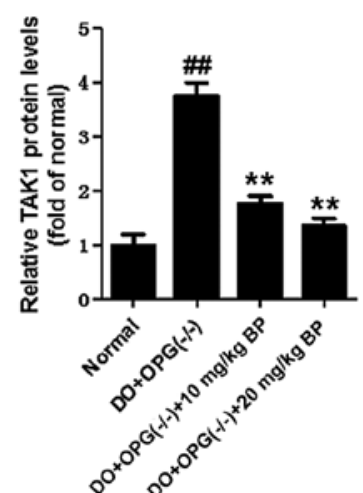

$\mathrm{H}$
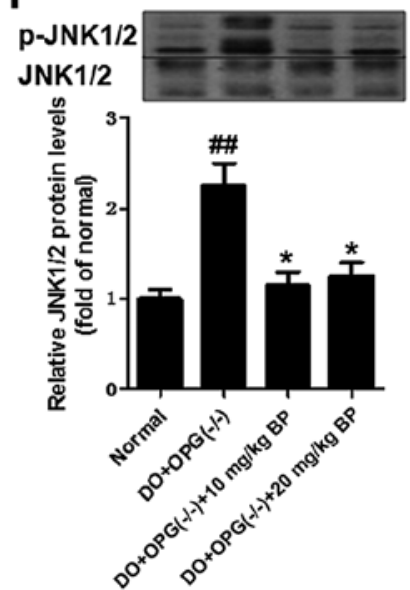

$\mathrm{DO}+20 \mathrm{mg} / \mathrm{kg}$ BP

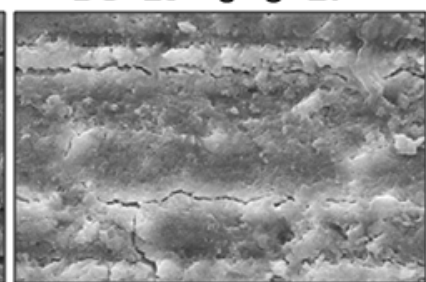

E
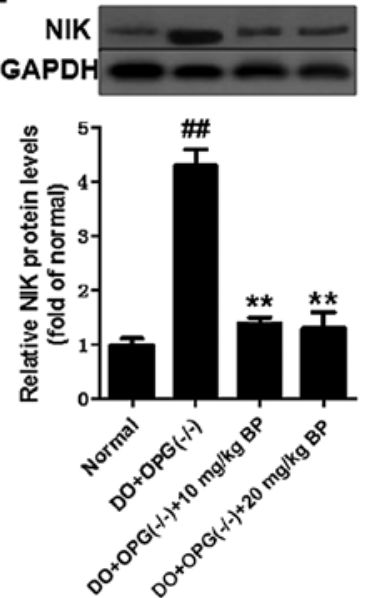

I
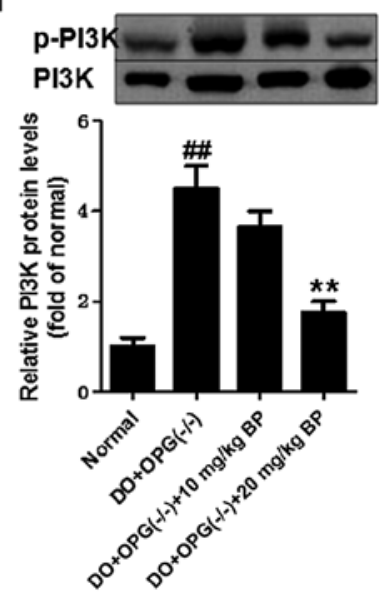

Figure 8. Bergapten suppresses the activation of the nuclear factor- $\mathrm{kB}(\mathrm{NF}-\mathrm{\kappa B})$, JNK/MAPK and AKT/mTOR signaling pathways in mice with high-fat dietinduced diabetes-related osteoporosis (DO) and with DO + OPG-KO. (A) Electron microscopic analysis of the bone surface structure. Arrows indicate the activation of osteoclasts. (B-F) Western blot analysis of the inhibitory effects of bergapten (BP) on the nuclear factor-kB (NF-kB) pathway. (G and H) Western blot analysis of the inhibitory effects of BP on the JNK/MAPK pathway. (I-K) Western blot analysis of the inhibitory effects of BP on the AKT/mTOR pathway. ${ }^{\# \#} \mathrm{p}<0.01$, vs. normal group; ${ }^{\mathrm{p}} \mathrm{p}<0.05$ and ${ }^{* * *} \mathrm{p}<0.01$, vs. DO and DO + OPG $(-/-)$ group. 


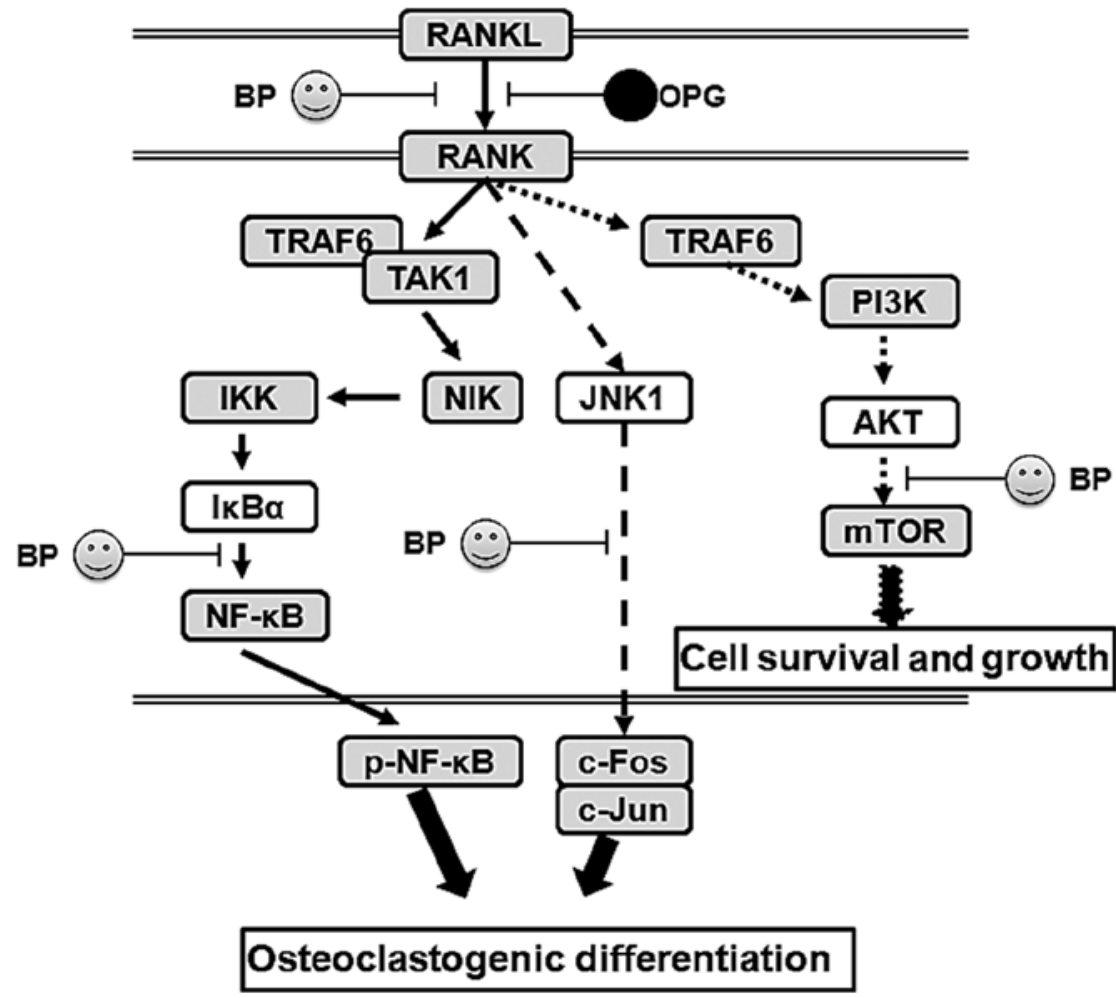

Figure 9. The possible molecular mechanisms of action of osteoprotegerin (OPG) in diabetes-related osteoporosis (DO) and the inhibitory effects of bergapten (BP) on the activation of the nuclear factor- $\mathrm{\kappa B}(\mathrm{NF}-\mathrm{\kappa B})$, JNK/MAPK and AKT/mTOR signaling pathways.

association with increased levels of phosphorylated $N F-\kappa B$, thus promoting the development of DO. We also investigated whether the anti-inflammatory agent, BP, exerts effects similar to those of OPG in mice with DO and with OPG-KO. Our data indicated that BP has the ability to indirectly or directly suppress the activation of the NF- $\mathrm{B}$, JNK and AKT/mTOR signaling pathways. It prevents trabecular bone injury and suppresses the loss of collagen.

In conclusion, in this study, we examined the protective effects of BP using mouse models of DO and and DO with OPG-KO. The DO and DO in conjunction with OPG-KO significantly promote the activation of the PI3K/AKT, JNK/MAPK and NF- $\kappa \mathrm{B}$ signaling pathways and increases the expression of related indicators, further promoting the release inflammatory cytokines, which damages trabecular bone structure and promotes osteoclastogenic differentiation.

\section{References}

1. Marcus R: Post-menopausal osteoporosis. Best Pract Res Clin Obstet Gynaecol 16: 309-327, 2002.

2. Mullin BH, Prince RL, Dick IM, Hart DJ, Spector TD, Dudbridge $\mathrm{F}$ and Wilson SG: Identification of a role for the ARHGEF3 gene in postmenopausal osteoporosis. Am J Hum Genet 82: 1262-1269, 2008

3. Reppe S, Refvem H, Gautvik VT, Olstad OK, Høvring PI, Reinholt FP, Holden M, Frigessi A, Jemtland R and Gautvik KM Eight genes are highly associated with BMD variation in postmenopausal Caucasian women. Bone: 604-612, 2010.

4. Jemtland R, Holden M, Reppe S, Olstad OK, Reinholt FP, Gautvik VT, Refvem H, Frigessi A, Houston B and Gautvik KM: Molecular disease map of bone characterizing the postmenopausal osteoporosis phenotype. J Bone Miner Res 26: 1793-1801, 2011.
5. Chao TH, Yu HN, Huang CC, Liu WS, Tsai YW and Wu WT: Association of interleukin-1 $\beta(-511 \mathrm{C} / \mathrm{T})$ polymorphisms with osteoporosis in postmenopausal women. Ann Saudi Med 30: 437-441, 2010.

6. Finkelstein JS, Brockwell SE, Mehta V, Greendale GA, Sowers MR, Ettinger B, Lo JC, Johnston JM, Cauley JA, Danielson ME and Neer RM: Bone mineral density changes during the menopause transition in a multiethnic cohort of women. J Clin Endocrinol Metab 93: 861-868, 2008.

7. Roy DK, Berry JL, Pye SR, Adams JE, Swarbrick CM, King Y, Silman AJ and O'Neill TW: Vitamin D status and bone mass in UK South Asian women. Bone 40: 200-204, 2007.

8. Bone HG, Greenspan SL, McKeever C, Bell N, Davidson M, Downs RW, Emkey R, Meunier PJ, Miller SS, Mulloy AL, et al: Alendronate and estrogen effects in postmenopausal women with low bone mineral density. Alendronate/Estrogen Study Group. J Clin Endocrinol Metab 85: 720-726, 2000.

9. Slemenda C, Longcope C, Peacock M, Hui S and Johnston CC: Sex steroids, bone mass, and bone loss. A prospective study of pre-, peri-, and postmenopausal women. J Clin Invest 97: 14-21, 1996.

10. Wolff I, van Croonenborg JJ, Kemper HC, Kostense PJ and Twisk JW: The effect of exercise training programs on bone mass: a meta-analysis of published controlled trials in pre- and postmenopausal women. Osteoporos Int 9: 1-12, 1999.

11. Clowes JA, Riggs BL and Khosla S: The role of the immune system in the pathophysiology of osteoporosis. Immunol Rev 208: 207-227, 2005

12. Cavalli L and Brandi ML: Age- and gender-related macro- and micro-architecture changes in bone structure and implications for treatment. Int J Clin Rheumatol 6: 359-369, 2011.

13. Sanders S and Geraci SA: Osteoporosis in postmenopausal women: Considerations in prevention and treatment: (Women's health series). South Med J 106: 698-706, 2013.

14. Hofbauer LC, Brueck CC, Singh SK and Dobnig H: Osteoporosis in patients with diabetes mellitus. J Bone Miner Res 22: 1317-1328, 2007.

15. Leidig-Bruckner G and Ziegler R: Diabetes mellitus a risk for osteoporosis? Exp Clin Endocrinol Diabetes 109 (Suppl 2:) S493-S514, 2001 
16. McLean RR: Proinflammatory cytokines and osteoporosis. Curr Osteoporos Rep 7: 134-139, 2009.

17. Huh JE, Lee WI, Kang JW, Nam D, Choi DY, Park DS, Lee SH and Lee JD: Formononetin attenuates osteoclastogenesis via suppressing the RANKL-induced activation of NF- $\kappa \mathrm{B}$, c-Fos, and nuclear factor of activated T-cells cytoplasmic 1 signaling pathway. J Nat Prod 77: 2423-2431, 2014.

18. Oh KW: Diabetes and Osteoporosis. Korean Diabetes J 33: 169-177, 2009 (In Korean).

19. Zheng M, Ge Y, Li H, Yan M, Zhou J and Zhang Y: Bergapten prevents lipopolysaccharide mediated osteoclast formation, bone resorption and osteoclast survival. Int Orthop 38: 627-634, 2014

20. Perez RM: Anti-inflammatory activity of compounds isolated from plants. ScientificWorldJournal 1: 713-784, 2001.

21. Peng X, Nie Y, Wu J, Huang Q and Cheng Y: Juglone prevents metabolic endotoxemia-induced hepatitis and neuroinflammation via suppressing TLR4/NF- $\mathrm{KB}$ signaling pathway in high-fat diet rats. Biochem Biophys Res Commun 462: 245-250, 2015.

22. Holstein P, Ellitsgaard N, Olsen BB and Ellitsgaard V: Decreasing incidence of major amputations in people with diabetes Diabetologia 43: 844-847, 2000.

23. Romero-Aroca P: Managing diabetic macular edema: The leading cause of diabetes blindness. World J Diabetes 2: 98-104, 2011.

24. Shoji T, Emoto M, Shinohara K, Kakiya R, Tsujimoto Y, Kishimoto $\mathrm{H}$, Ishimura $\mathrm{E}$, Tabata $\mathrm{T}$ and Nishizawa $\mathrm{Y}$ : Diabetes mellitus, aortic stiffness, and cardiovascular mortality in end-stage renal disease. J Am Soc Nephrol 12: 2117-2124, 2001.

25. Schneider PA, Caps MT, Ogawa DY and Hayman ES Intraoperative superficial femoral artery balloon angioplasty and popliteal to distal bypass graft: an option for combined open and endovascular treatment of diabetic gangrene. J Vasc Surg 33: 955-692, 2001.
26. Balkau B, Shipley M, Jarrett RJ, Pyörälä K, Pyörälä M, Forhan A and Eschwège E: High blood glucose concentration is a risk factor for mortality in middle-aged nondiabetic men. 20-year follow-up in the Whitehall Study, the Paris Prospective Study, and the Helsinki Policemen Study. Diabetes Care 21: 360-367. 1998.

27. Sumner CJ, Sheth S, Griffin JW, Cornblath DR and Polydefkis M: The spectrum of neuropathy in diabetes and impaired glucose tolerance. Neurology 60: 108-111, 2003.

28. Kim C, Newton KM and Knopp RH: Gestational diabetes and the incidence of type 2 diabetes: a systematic review. Diabetes Care 25: 1862-1868, 2002

29. Moran C, Münch G, Forbes JM, Beare R, Blizzard L, Venn AJ, Phan TG, Chen J and Srikanth V: Type 2 diabetes, skin autofluorescence, and brain atrophy. Diabetes 64: 279-283, 2015.

30. Zinman B, Lachin JM and Inzucchi SE: Empagliflozin, cardiovascular outcomes, and mortality in type 2 diabetes. N Engl J Med 374: 1094, 2016.

31. Wu YY, Xiao E and Graves DT: Diabetes mellitus related bone metabolism and periodontal disease. Int J Oral Sci 7: 63-72, 2015.

32. Li R, Xu W, Luo S, Xu H, Tong G, Zeng L, Zhu D and Weng J: Effect of exenatide, insulin and pioglitazone on bone metabolism in patients with newly diagnosed type 2 diabetes. Acta Diabetol 52: 1083-1091, 2015.

33. Wu QQ, Xu M, Yuan Y, Li FF, Yang Z, Liu Y, Zhou MQ, Bian ZY, Deng W, Gao L, et al: Cathepsin B deficiency attenuates cardiac remodeling in response to pressure overload via TNF- $\alpha / \mathrm{ASK} 1 /$ JNK pathway. Am J Physiol Heart Circ Physiol 308: H1143-H1154, 2015.

34. Weston CR and Davis RJ: The JNK signal transduction pathway. Curr Opin Cell Biol 19: 142-149, 2007. 